\title{
Hypohamiltonian graphs and their crossing number
}

\author{
Carol T. Zamfirescu \\ Faculty of Mathematics \\ TU Dortmund \\ Dortmund, Germany \\ czamfirescu@gmail.com
}

Submitted: Apr 28, 2012; Accepted: Sep 18, 2012; Published: Oct 25, 2012

Mathematics Subject Classifications: 05C45

\begin{abstract}
We prove that for every $k \geqslant 0$ there is an integer $n_{0}(k)$ such that, for every $n \geqslant n_{0}$, there exists a hypohamiltonian graph which has order $n$ and crossing number $k$.
\end{abstract}

Keywords: Hypohamiltonian graph; crossing number

\section{Introduction}

Throughout this paper all graphs will be undirected, finite, connected, and without loops or multiple edges. For a graph $G$, we denote by $V(G)$ its vertex set and by $E(G)$ its edge set. The path $P$ with $\{u v\}=E(P)$ will also be denoted by $u v$. A graph $G$ is hypohamiltonian if $G$ is not hamiltonian but for any $v \in V(G)$ the graph $G-v$ is hamiltonian. Denote the set of all hypohamiltonian graphs by $\mathfrak{P}$. For an overview, see the survey of Holton and Sheehan [8]. Not included therein are recent results concerning the planar case, due to the author and Zamfirescu [17], Araya and Wiener [3, 15], the author [16], and Jooyandeh, McKay, Östergård, Pettersson, and the author [9].

A drawing of a graph $G$ is an injective mapping $f$ that assigns to each vertex a point in the plane and to each edge $u v$ a Jordan arc (i.e. a homeomorphic image of a closed interval) connecting $f(u)$ and $f(v)$, not passing through the image of any other vertex. For simplicity, the arc assigned to $u v$ is called an edge of the drawing, and if this leads to no confusion, it is also denoted by $u v$. We assume that no three edges have an interior point in common, and if two edges share an interior point $p$, then they cross at $p$. We also assume that any two edges of a drawing have only a finite number of crossings (i.e. common interior points). A common endpoint of two edges does not count as a crossing. 
The crossing number of $G$, denoted by $\operatorname{cr}(G)$, is the minimum number of edge crossings for all possible drawings of $G$. An overview of results concerning the crossing number can be found in Székely's paper [11] and the unpublished manuscript [10] by Richter and Salazar.

It is our aim in this note to study the crossing number of certain hypohamiltonian graphs. Little seems to be known. We shall use the family of generalized Petersen graphs $\mathrm{GP}(n, k)$ introduced by Coxeter [5]. A graph in $\operatorname{GP}(n, k)$ has vertex set $\left\{u_{1}, \ldots, u_{n}, v_{1}, \ldots, v_{n}\right\}$ and edge set $\bigcup_{i=1}^{n}\left\{u_{i} u_{i+1}, u_{i} v_{i}, v_{i} v_{i+k}\right\}$, the indices being taken modulo $n$, and $1 \leqslant k \leqslant\lfloor(n-1) / 2\rfloor$. Alspach [2] showed that $\operatorname{GP}(n, k)$ is non-hamiltonian if and only if $n \equiv 5(\bmod 6)$ and $k=2$. We call this particular subfamily $\mathcal{F}$. Bondy [4] showed that $\mathcal{F} \subset \mathfrak{P}$.

The Petersen graph $P=\operatorname{GP}(5,2) \in \mathcal{F}$ has crossing number 2. The proof this wellknown fact is short. Firstly, draw $P$ with two crossings (see Fig. 1). Secondly, prove that it is impossible to draw the Petersen graph with less than two crossings. One can do this with the following argument based on Euler's formula (see for instance [11]). Denote the girth, size, order of a graph $G$ by $g, m, n$, respectively. Then we have

$$
\operatorname{cr}(G) \geqslant\left\lceil m-\frac{g(n-2)}{g-2}\right\rceil .
$$

For $P, g=5, m=15, n=10$; thus $(\dagger)$ yields $\operatorname{cr}(P) \geqslant 2$.

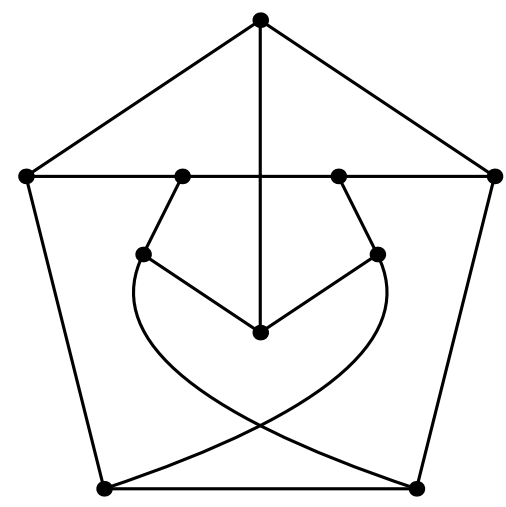

Figure 1: Petersen's graph drawn with two edge crossings.

Exoo, Harary, and Kabell [6] showed that $\operatorname{cr}(\operatorname{GP}(n, 2))=3$ when $n$ is odd and at least 7. Therefore, all other members of $\mathcal{F}$ have crossing number 3.

In 2011, it was shown by Araya and Wiener [15] that there exist planar hypohamiltonian graphs of order $n$ for every $n \geqslant 76$. This bound was recently improved to 42 by Jooyandeh, McKay, Östergård, Pettersson, and the author [9]. The smallest hypohamiltonian graph is the Petersen graph, which has crossing number 2. All non-planar hypohamiltonian graphs constructed (explicitly) in the literature seem to have crossing number at least 2 , and in fact for few of them the crossing number has been computed explicitly. 
For a vertex $x$, we denote by $N(x)$ the set of vertices which are joined by an edge to $x$. We will call a 4-cycle or a quadrilateral face cubic, if all of its vertices are cubic. For a set $A \subset V(G)$ we denote by $G[A]$ the graph spanned by $A$ in $G$.

\section{Result}

We require a construction method introduced by Thomassen [14]. Let $G \in \mathfrak{P}$ contain a 4-cycle $a b c d a=C$. We delete the edges $a b$ and $c d$, add two new vertices $a^{\prime}$ and $d^{\prime}$, and add the edges $a^{\prime} d^{\prime}, a a^{\prime}, d d^{\prime}, a^{\prime} c, d^{\prime} b$. Denote the resulting graph by $G_{C}^{\star}$. Whenever the choice of $C$ is clear, we simply write $G^{\star}$. For an illustration of a case where $G$ is planar, see Fig. 2.

Lemma 1. If $G \in \mathfrak{P}$ contains a cubic 4-cycle $C$, then $G_{C}^{\star} \in \mathfrak{P}$. If $G$ is planar, then $\operatorname{cr}\left(G_{C}^{\star}\right)=1$.

Thomassen mentions in [14] the first part of the above statement, but gives no proof. Therefore, we choose to show here both parts.

Proof. Notice that the 4-cycle $a b c d a=C$ is chordless, as it is cubic and $G$ is 3-connected. Assume $G^{\star}$ contains a hamiltonian cycle $H$. There are four essentially different possibilities for $H$ to visit $a^{\prime}$ and $d^{\prime}$.

(i) $c a^{\prime} d^{\prime} b \subset H$. Replacing $c a^{\prime} d^{\prime} b$ with $c b$ we obtain a hamiltonian cycle in $G$, a contradiction.

(ii) $c a^{\prime} a \subset H$. Then $b d^{\prime} d \subset H$. Replace either $c a^{\prime} a$ with $c b$ and $b d^{\prime} d$ with $a d$ or $c a^{\prime} a$ with $c d$ and $b d^{\prime} d$ with $a b$. One of these replacements yields a hamiltonian cycle, which leads to contradiction.

(iii) $a a^{\prime} d^{\prime} d \subset H$. Then $b c \subset H$. Replacing $a a^{\prime} d^{\prime} d$ with $a d$ we obtain a hamiltonian cycle in $G$, a contradiction.

(iv) $c a^{\prime} d^{\prime} d \subset H$. Then $b c a^{\prime} d^{\prime} d a \subset H$ and replacing $c a^{\prime} d^{\prime} d$ by $c d$ yields a hamiltonian cycle in $G$, a contradiction.

Hence, $G^{\star}$ is non-hamiltonian.

Put $n=|V(G)|$ and $n^{\star}=\left|V\left(G^{\star}\right)\right|$. We now show that for each $v \in V\left(G^{\star}\right)$ there exists an $\left(n^{\star}-1\right)$-cycle $H_{v}^{\star}$ in $G^{\star}-v$. Consider $v \in V(G) \backslash\{a, b, c, d\}$ and the $(n-1)$-cycle $H_{v}$ in $G-v$. Observe that $H_{v}$ must contain two opposing edges of $C$. There are two cases to consider.

(i) If $a b \subset H$ and $c d \subset H$, then either replace $a b$ with $a a^{\prime} c$ and $c d$ with $b d^{\prime} d$ or replace $a b$ with $a a^{\prime} d^{\prime} d$ and $c d$ with $c b$. As above, one of these replacements yields a hamiltonian cycle.

(ii) If $a d \subset H$ and $b c \subset H$, then in case $c d \not \subset H$ replace $a d$ with $a a^{\prime} d^{\prime} d$, and in case $c d \subset H$ replace $c d$ by $c a^{\prime} d^{\prime} d$.

We obtain $H_{b}^{\star}$ from $H_{b}$ and $H_{a}^{\star}$ from $H_{a}$ by replacing $d c$ with $d d^{\prime} a^{\prime} c$. Analogously we get $H_{c}^{\star}$ and $H_{d}^{\star}$. For $H_{a^{\prime}}^{\star}$ consider $H_{d}$ and replace $a b$ with $a d d^{\prime} b$. Analogously we get $H_{d^{\prime}}^{\star}$.

This shows that $G^{\star} \in \mathfrak{P}$. 
Now suppose $G$ is planar. $G^{\star}$ contains a graph $K$ homeomorphic to $K_{3,3}$, where $V(K)=\left\{a, c, d^{\prime}, a^{\prime}, b, d\right\}$. Thus, by Kuratowski's Theorem, $G^{\star}$ is non-planar. It is easy to draw $G^{\star}$ in the plane with exactly one crossing (for an example, see Fig. 2).

Lemma 2. There exists a hypohamiltonian graph with crossing number 1.

Proof. Apply Lemma 1 to a planar cubic hypohamiltonian graph containing a quadrilateral face. For instance we can take the 76-vertex graph $Q$ of Fig. 2 constructed by the author [16]. The resulting graph $Q^{\star}$ is shown in Fig. 2. Using the second part of Lemma 1, we obtain the statement.
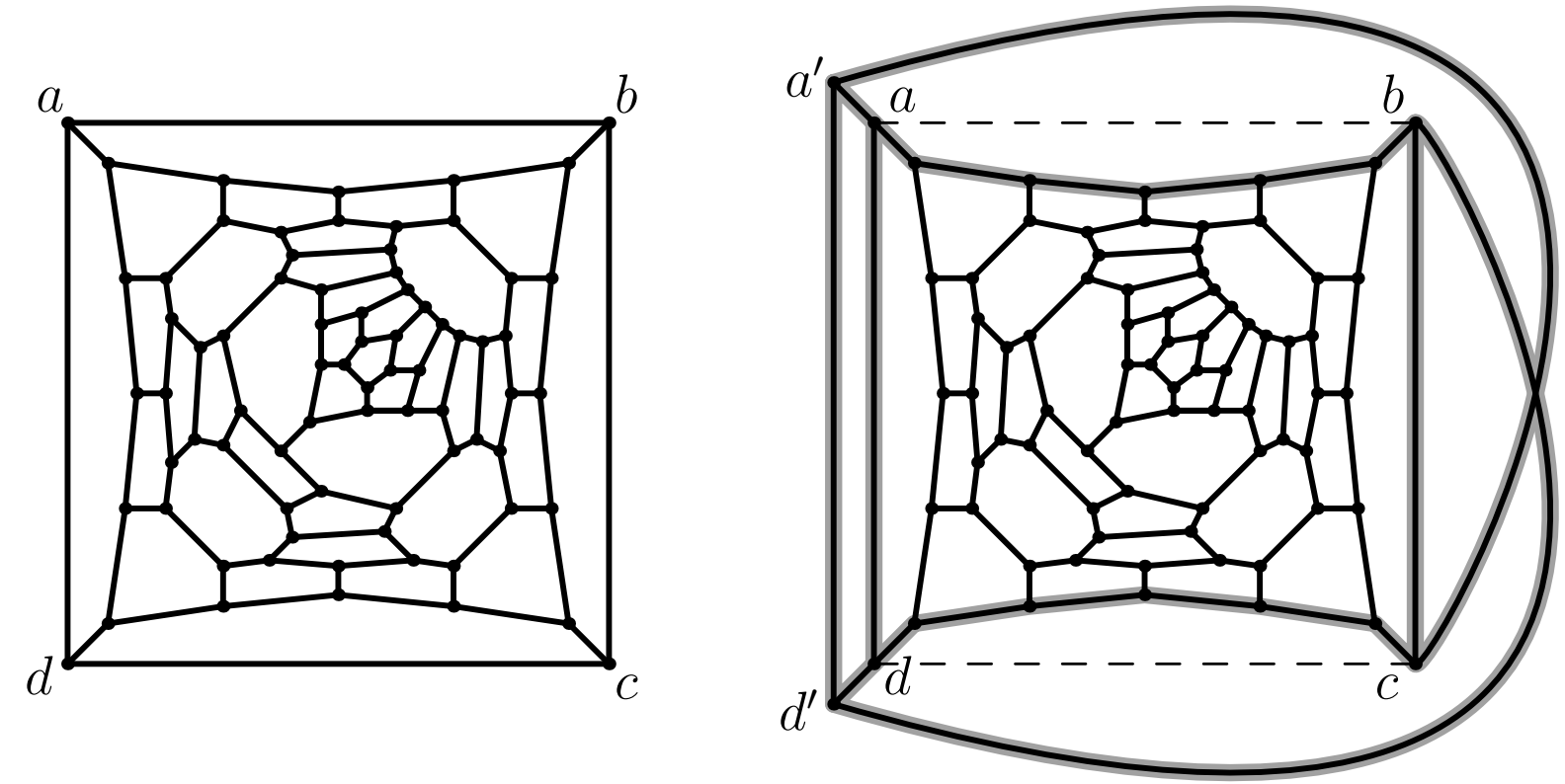

Figure 2: On the left, a planar cubic hypohamiltonian graph $Q$ is shown; on the right, $Q^{\star}$ is shown. $Q^{\star}$ has crossing number 1 . The grey edges in $Q^{\star}$ show a subgraph homeomorphic to $K_{3,3}$.

Consider graphs $G$ and $G^{\prime}$ containing cubic vertices $x$ and $x^{\prime}$, respectively. Denote by $G_{x} G_{x^{\prime}}^{\prime}$ one of the graphs obtained from $G-x$ and $G^{\prime}-x^{\prime}$ by identifying the vertices in $N(x)$ with those in $N\left(x^{\prime}\right)$ using a bijection (see Fig. 3). The next lemma (Lemma 3) is due to Thomassen [12], while Lemma 4 is easy to show and its proof is left to the reader. For an illustration of Lemma 4, see Fig. 3.

Lemma 3. Let $G, G^{\prime} \in \mathfrak{P}$ each contain a cubic vertex, say $x$ and $x^{\prime}$, respectively. Then $G_{x} G_{x^{\prime}}^{\prime} \in \mathfrak{P}$.

Lemma 4. Assume that $G$ and $G^{\prime}$ contain cubic vertices $x \in V(G)$ and $x^{\prime} \in V\left(G^{\prime}\right)$, respectively, such that $x \notin \mathcal{U}$, where $\mathcal{U}$ is the set of vertices of all crossing edges in a drawing of $G$ with $\operatorname{cr}(G)$ crossings, and $G^{\prime}$ is planar. Then $\operatorname{cr}\left(G_{x} G_{x^{\prime}}^{\prime}\right)=\operatorname{cr}(G)$. 


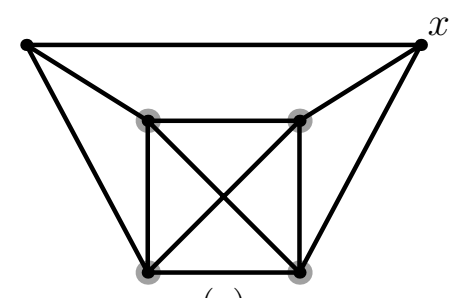

(a)

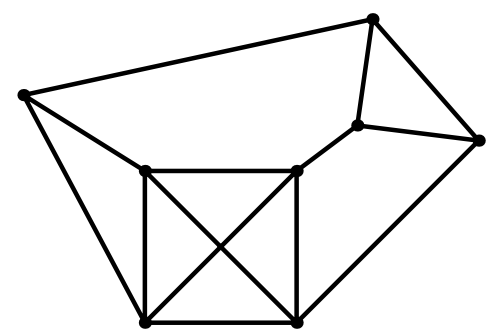

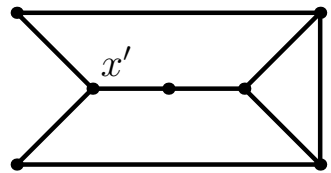

(b)

(c)

Figure 3: (a) shows a non-planar graph $G$. The vertices of $\mathcal{U}$ are marked with a grey boundary. (b) depicts a planar graph $G^{\prime}$. (c) illustrates Lemma 4: $\operatorname{cr}\left(G_{x} G_{x^{\prime}}^{\prime}\right)=\operatorname{cr}(G)=1$.

Lemma 5. For any $k \geqslant 0$ there exists a hypohamiltonian graph which has crossing number $k$.

Proof. For $k=0$, consider a planar cubic hypohamiltonian graph such as the graph $Q$ of Fig. 2. Put $Q^{1}=Q^{\star}$ (see Fig. 2). In $Q$ minus the edges $a b$ and $c d$, denote the shortest path between $a$ and $b$ by $S$ and the shortest path between $c$ and $d$ by $T$. Consider

$$
J=Q^{\star}\left[\left\{a, a^{\prime}, b, c, d, d^{\prime}\right\}\right] \cup S \cup T,
$$

marked with grey edges in Fig. 2. Consider a cubic vertex $v \in V\left(Q^{\star}\right)$ with $N(v) \cap J=\emptyset$. Take two copies $Q^{\prime}, Q^{\prime \prime}$ of $Q^{\star}$, the corresponding subgraphs $J^{\prime}, J^{\prime \prime}$, and the corresponding vertices $v^{\prime}, v^{\prime \prime}$. Consider $Q^{2}=Q_{v^{\prime}}^{\prime} Q_{v^{\prime \prime}}^{\prime \prime}$.

This graph contains two graphs homeomorphic to $K_{3,3}$, namely $J^{\prime}$ and $J^{\prime \prime}$, and $J^{\prime} \cap$ $J^{\prime \prime}=\emptyset$. Since $\operatorname{cr}\left(K_{3,3}\right)=1$, we have $\operatorname{cr}\left(Q^{2}\right)=2$. Now construct in the same manner $Q^{k}=Q_{w}^{k-1} Q_{v}^{1}$ (where $w \in V\left(Q^{k-1}\right)$ is a cubic vertex such that $N(w)$ does not meet any subgraph isomorphic to $J$ ), yielding $\operatorname{cr}\left(Q^{k}\right)=k$. We may iterate this ad infinitum, as in every step many cubic vertices are introduced.

Theorem 6. For every $k \geqslant 0$ there is an integer $n_{0}(k)$ such that, for every $n \geqslant n_{0}$, there exists a hypohamiltonian graph which has order $n$ and crossing number $k$.

Proof. Let $Q^{k}$ be the hypohamiltonian graph with crossing number $k$ constructed in the proof of Lemma 5. It has plenty of cubic vertices. Now choose a cubic vertex $v \in V\left(Q^{k}\right)$ no neighbour of which belongs to any of the used subgraphs isomorphic to $J$. For every $n \geqslant 42$ there exists a planar hypohamiltonian graph $H^{n}$ of order $n$ [9]. By a result of Thomassen [13], every planar hypohamiltonian graph contains a cubic vertex. Thus, for 
every $n$ the graph $H^{n}$ contains a cubic vertex $w$. We apply Lemmas 3 and 4 to $Q^{k}$ and $H^{n}$, obtaining the graph $Q_{v}^{k} H_{w}^{n}$. Providing the family $\left\{Q_{v}^{k} H_{w}^{n}\right\}_{n=42}^{\infty}$ yields the statement.

\section{Remarks on $\mathfrak{c}_{k}, \mathfrak{h}_{k}$, and the cubic case}

Let $\mathfrak{c}_{k}$ be the order of the smallest hypohamiltonian graph with crossing number $k$, and let $\mathfrak{h}_{k}$ denote the minimum number such that there exists a hypohamiltonian graph of order $n$ and crossing number $k$ for every $n \geqslant \mathfrak{h}_{k}$.

An upper bound for $\mathfrak{c}_{0}$ is a direct consequence of a result from [9], where it is shown that there exists a planar hypohamiltonian graph of order 40 . We now prove that $\mathfrak{c}_{1} \leqslant 46$. By applying a method of Thomassen [14], called in [15] "Thomassen operation" and denoted by $\mathrm{Th}$, in [9] it is shown that there exists a planar hypohamiltonian graph $W$ of order 44 containing a quadrilateral cubic face. By Lemma $1, W^{\star}$ is a hypohamiltonian graph of order 46 and crossing number 1 . We recall that the Petersen graph $P$ has crossing number 2 , so $\mathfrak{c}_{2}=10$ (as $P$ is the smallest hypohamiltonian graph, see e.g. [7]). Moreover, $\operatorname{cr}(\operatorname{GP}(11,2))=3$, see [6]. In summary, we have

$$
18 \leqslant \mathfrak{c}_{0} \leqslant 40, \quad 18 \leqslant \mathfrak{c}_{1} \leqslant 46, \quad \mathfrak{c}_{2}=10, \quad \text { and } \quad 13 \leqslant \mathfrak{c}_{3} \leqslant 22,
$$

where the lower bounds on $\mathfrak{c}_{0}$ and $\mathfrak{c}_{1}$ can be computed by using the list of small hypohamiltonian graphs provided by Aldred, McKay and Wormald [1] and applying ( $\dagger$ ): all seven hypohamiltonian graphs on fewer than 18 vertices have crossing number at least 2.

By [9], we have $\mathfrak{h}_{0} \leqslant 42$. For $k=1$ denote by $H^{n}$ the hypohamiltonian graph of order $n$ used to show the aforementioned result from [9]. By applying the Thomassen operation to $H^{n}$ we obtain a planar hypohamiltonian graph $\operatorname{Th}\left(H^{n}\right)$ of order $n+4$ with a cubic quadrilateral face. Via Lemma 1 we obtain the family $\left\{\left(\operatorname{Th}\left(H^{n}\right)\right)^{\star}\right\}_{n=42}^{\infty}$ with $\left|V\left(\left(\operatorname{Th}\left(H^{n}\right)\right)^{\star}\right)\right|=n+6$. As mentioned before, we have $\operatorname{cr}(P)=2$ and $\operatorname{cr}(\operatorname{GP}(11,2))=3$, so by applying Lemma 3 we obtain the families $\left\{P_{x} H_{y}^{n}\right\}_{n=42}^{\infty}$ and $\left\{\operatorname{GP}(11,2)_{x} H_{y}^{n}\right\}_{n=42}^{\infty}$. All of the above shows that

$$
\mathfrak{h}_{0} \leqslant 42, \quad \mathfrak{h}_{1} \leqslant 48, \quad \mathfrak{h}_{2} \leqslant 47, \quad \text { and } \quad \mathfrak{h}_{3} \leqslant 59 .
$$

The author [16] showed that there exists a planar cubic hypohamiltonian graph $H^{n}$ of order $n$ for every $n \geqslant 76$. Each $H^{n}$ contains a (unique) quadrilateral face, and each member of the family $\left\{\left(H^{n}\right)^{\star}\right\}_{n=76}^{\infty}$ has crossing number 1 . Therefore, for every even $n \geqslant 78$ there exists a cubic hypohamiltonian graph which has order $n$ and crossing number 1 .

\section{References}

[1] R. E. L. Aldred, B. D. McKay, and N. C. Wormald. Small hypohamiltonian graphs. J. Combin. Math. Combin. Comput., 23:143-152, 1997. 
[2] B. R. Alspach. The Classification of Hamiltonian Generalized Petersen Graphs. J. Combin. Theory Ser. B, 34:293-312, 1983.

[3] M. Araya and G. Wiener. On cubic planar hypohamiltonian and hypotraceable graphs. Electron. J. Combin., \#P85:1-11, 2011.

[4] J. A. Bondy. Variations on the hamiltonian theme. Canad. Math. Bull., 15:57-62, 1972.

[5] H. S. M. Coxeter. Self-Dual Configurations and Regular Graphs. Bull. Amer. Math. Soc., 56:413-455, 1950.

[6] G. Exoo, F. Harary, and J. Kabell. The crossing numbers of some generalized Petersen graphs. Math. Scand., 48:184-188, 1981.

[7] J. C. Herz, J. J. Duby, and F. Vigué. Recherche systématique des graphes hypohamiltoniens. In Theory of Graphs: International Symposium, Rome, 1966, pages 153-159. Gordon and Breach, New York, and Dunod, Paris, 1967.

[8] D. A. Holton and J. Sheehan. Chapter 7: Hypohamiltonian graphs. In The Petersen Graph, Cambridge University Press, New York, 1993.

[9] M. Jooyandeh, B. McKay, P. Östergård, V. Pettersson, and C. T. Zamfirescu. Planar hypohamiltonian graphs on 40 vertices. Work in progress.

[10] R. B. Richter and G. Salazar. Crossing numbers. http://www.math.uwaterloo.ca/ brichter/pubs/June2008.pdf

[11] L. A. Székely. A successful concept for measuring non-planarity of graphs: the crossing number. Discrete Math., 276:331-352, 2004.

[12] C. Thomassen. Hypohamiltonian and hypotraceable graphs. Discrete Math., 9:91-96, 1974.

[13] C. Thomassen. Hypohamiltonian graphs and digraphs. In Theory and applications of graphs: Proceedings, Western Michigan Univ., Kalamazoo, MI, 1976, volume 642 of Lecture Notes in Mathematics, pages 557-571. Springer, 1978.

[14] C. Thomassen. Planar cubic hypohamiltonian and hypotraceable graphs. J. Combin. Theory Ser. B, 30:36-44, 1981.

[15] G. Wiener and M. Araya. On planar hypohamiltonian graphs. J. Graph Theory, 67(1):55-68, 2011.

[16] C. T. Zamfirescu. On hypohamiltonian and almost hypohamiltonian graphs. Work in progress.

[17] C. T. Zamfirescu and T. I. Zamfirescu. A planar hypohamiltonian graph with 48 vertices. J. Graph Theory, 55(4):338-342, 2007. 